\title{
Efecto del tiempo de superpulso de soldadura sobre la evolución microestructural de un acero de bajo carbono
}

\author{
Effect of weld superpulse time on the \\ microstructural evolution of a \\ low carbon steels
}

\author{
Agustín Gualco $^{1,2}$, Franco Raad ${ }^{1}$, \\ Sebastián Zappa ${ }^{1,2}$
}

\footnotetext{
${ }^{1}$ Instituto de Investigación en Soldadura- FI-UNLZ CP: 1832, Lomas de Zamora, Buenos Aires, Buenos Aires, Argentina ${ }^{2}$ Consejo Nacional de Investigaciones Científicas y Técnicas CP: 1425, Buenos Aires, Buenos Aires, Argentina e-mail: agustingualco@yahoo.com.ar
}

\section{RESUMEN}

El tipo de arco Súper Pulso fue desarrollado para proporcionar una solución a los cordones de pasada de raíz de juntas a tope, en el proceso de soldadura semiautomático con protección gaseosa (GMAW). A diferencia de la soldadura por pulsos estándar, el Súper Pulso utiliza una secuencia de diferentes formas de onda de pulso para crear una forma y aspecto de cordón similar al proceso GTAW. Utiliza alto amperaje en la fase primaria para mejorar la penetración y un bajo amperaje en la segunda fase para disminuir el calor aportado. Se utiliza la transferencia de spray en la fase primaria para mejorar la penetración y arco corto en la fase secundaria para enfriar el baño de soldadura dando menor transferencia de calor y menor distorsión al material de base. En la literatura hay poca información respecto a la relación entre las variables del Súper Pulso y las características microestructurales del metal depositado. El objetivo de este trabajo fue evaluar la influencia de los tiempos de cada fase en modo Súper Pulso, sobre las características geométricas del cordón y la solidificación en depósitos de soldadura de aceros con bajo contenido de carbono. Para tal fin, se soldaron cordones sobre una chapa IRAM - IAS U500 42 - F24 variando el tiempo de fases del Súper Pulso. Sobre cortes transversales se determinó la geometría de los cordones y sobre cortes longitudinales la microestructura, las características de las zonas fundidas y afectadas. Resultados preliminares mostraron que la geometría del cordón y la solidificación estuvieron fuertemente influenciadas por los parámetros del pulso.

Palabras claves: Soldadura pulsada, tiempos entre pulsos, acero al carbono

\section{ABSTRACT}

The Super Pulse arc type was developed to provide a solution to butt root pass beads in the semi-automatic welding process with gas protection (GMAW). Unlike standard pulse welding, the Super Pulse uses a sequence of different pulse waveforms to create a cord shape and appearance similar to the GTAW process. It uses higher amperage in the primary phase to improve penetration and low amperage in the second phase to reduce the heat input. Spray transfer is used in the primary phase to improve penetration and short arc in the secondary phase to cool the solder bath giving less heat transfer and less distortion to the base material. In the literature there is little information regarding the relationship between the Super Pulse variables and the microstructural characteristics of the deposited metal. The objective of this work was to evaluate the influence of the times of each phase in Super Pulse mode, on the geometric characteristics of the cord and the solidification in weld deposits of low carbon steels. For this purpose, strands should be welded on an IRAM - IAS U500 42 - F24 plate by varying the phase time of the Super Pulse. In cross sections, the geometry of the bead was determined and in longitudinal sections, the microstructure and the characteristics of the molten and affected areas were determined. Preliminary results showed that bead geometry and solidification were strongly influenced by pulse parameters.

Keywords: pulse welding, time of pulses, low carbon steel. 


\section{INTRODUCCIÓN}

El sistema de soldadura por arco Superpulso fue desarrollado para, principalmente, maximizar la productividad en chapas de espesores finos y obtener la geometría de cordón adecuada en soldaduras de pasada de raíz para chapas gruesas. Es un nuevo proceso de soldadura que pretende sustituir al proceso TIG para atender la demanda de productividad cuando es esencial el control de aporte de calor. El control preciso del calor aportado, elimina la distorsión generando una muy buena terminación superficial [1-5]. El sistema Superpulso se puede considerar una alternancia programada entre dos ajuste. Los intervalos de tiempo se determinan mediante los ajustes efectuados en los parámetros de tiempo de fase primario / secundario. De esta manera es posible obtener un primer ciclo caliente en modo spray convencional y un segundo ciclo frío en modo de arco corto. La Figura 1 muestra un esquema del proceso y la variación de la corriente en función del tiempo.

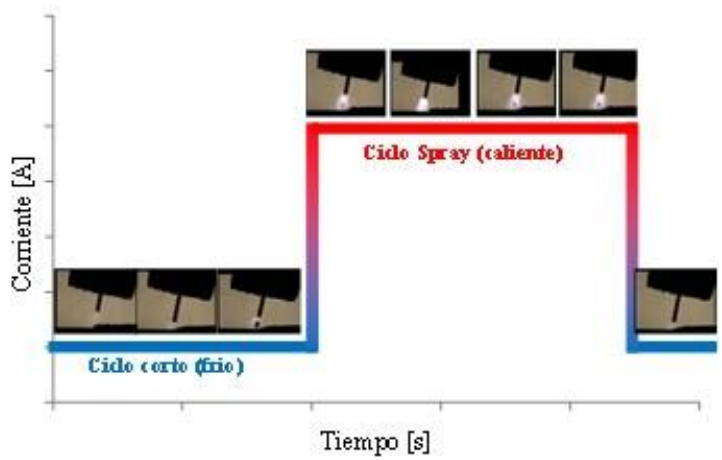

Figura 1: Esquema del proceso de soldadura y variación de la corriente en función del tiempo.

Las principales ventajas del Sistema Superpulso son:

- Menos sensibilidad a variaciones en la separación de la raíz.

- Mejor control del baño de soldadura durante la soldadura sobre plano.

- Mejor control de la penetración y del perfil de la penetración.

- Menor sensibilidad a la conducción desigual del calor.

- Control total sobre el calor aportado.

El objetivo de este trabajo fue estudiar el efecto de los tiempos de los ciclos calientes y fríos (spray/corto) sobre la geometría del cordón, el perfil de penetración, la dilución, el modo de solidificación y la dureza en cordones de soldadura de acero al carbono.

\section{MATERIALES Y MÉTODOS}

Se soldaron 4 cordones de soldadura (tipo bead on plate) modificando únicamente los tiempos de los ciclos calientes y fríos. Los materiales base fueron cortes de chapas de acero comercial de bajo contenido de carbono de 100 x 200 x $12,7 \mathrm{~mm}$ y el consumible empleado fue un alambre macizo que responde a la norma AWS A5.18-05 [6] con clasificación ER70S-6. El gas de protección utilizado fue $\mathrm{CO}_{2}$. La distancia pico de contacto-pieza y la posición de la torcha estuvo comprendida entre 15 y $20 \mathrm{~mm}$ y 75 y $80^{\circ}$, respectivamente. En todos los casos los parámetros eléctricos y la velocidad de soldadura se mantuvieron constantes. La Tabla 1 muestra la identificación de los cupones y los valores promedios de los parámetros eléctricos reportados por la máquina. La Figura 2 muestra los ciclos de corriente utilizados para cada condición.

Tabla 1: Identificación de los cupones y tiempos de ciclos.

\begin{tabular}{c|c|c|c|c|c|c}
\hline \multirow{2}{*}{ CUPON } & T SPRAY & T CORTO & TENSIÓN & CORRIENTE & V SOLDADURA & CALOR APORTADO \\
\cline { 2 - 7 } & [segundos] & [segundos] & {$[\mathrm{V}]$} & {$[\mathrm{A}]$} & {$[\mathrm{mm} / \mathrm{s}]$} & {$[\mathrm{kJ} / \mathrm{mm}]$} \\
\hline SC1 & 0,1 & 1,0 & 21,5 & 154 & 5,3 & 0,62 \\
\hline SC2 & 0,2 & 0,8 & 21,7 & 160 & 5,3 & 0,66 \\
\hline SC3 & 0,3 & 0,6 & 23,0 & 174 & 5,3 & 0,76 \\
\hline SC4 & 0,4 & 0,4 & 23,2 & 177 & 5,3 & 0,78 \\
\hline
\end{tabular}

Siendo: T SPRAY: Tiempo del ciclo en transferencia spray; T CORTO: Tiempo del ciclo en transferencia corto circuito; V SOLDADURA: Velocidad de soldadura. 


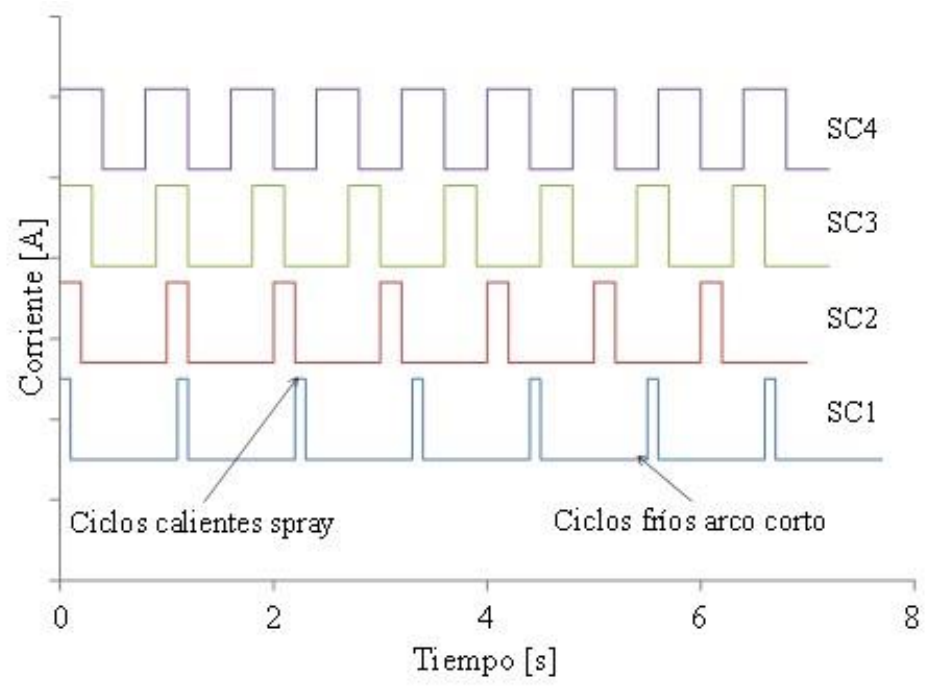

Figura 2: Ciclos de corriente vs tiempo para cada condición soldada.

Una vez soldados los cordones se realizó una inspección visual, se tomaron fotos superficiales y se realizaron cortes transversales y longitudinales para la caracterización macro y microestructural. Se midió el ancho y el sobre espesor en muestras transversales y el perfil de penetración en muestras longitudinales de acuerdo a lo mostrado en la Figura 3. Además, se midió la distancia entre valles, siendo ésta la distancia entre las zonas de mayor penetración. Finalmente, se observó el modo de solidificación mediante microscopia óptica, midiendo el tamaño del grano columnar y se realizaron barridos de microdureza Vickers con $1 \mathrm{~kg}$ de carga cada $0,25 \mathrm{~mm}$ desde la superficie.

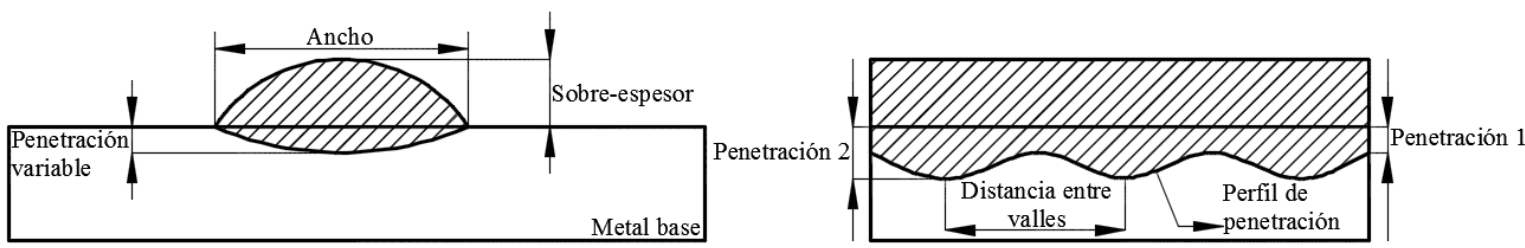

Figura 3: Geometría del cordón transversal y longitudinal.

\section{RESULTADOS}

No se observaron discontinuidades mediante la inspección visual. La Figura 4 muestra imágenes superficiales de los cordones soldados. En dicha figura se puede apreciar, en forma cualitativa, que el nivel de salpicaduras disminuye de SC1 a SC4, es decir, al disminuir el tiempo del ciclo de corto circuito de 0,1 a 0,4. Además, se observa que el ancho de las ondulaciones (ripples) de soldadura aumenta de SC1 a SC4 y que la forma de los mismos pasa de ser de forma circular a elíptica para las probetas soldadas con mayor tiempo de spray.

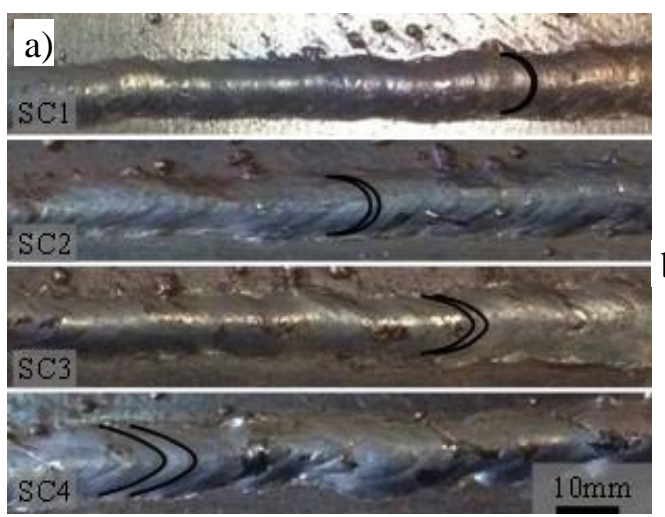

b) Formas de las piletas líquidas

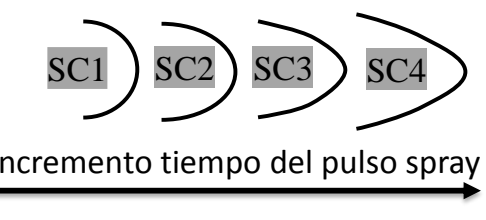

Figura 4:a) Apariencia superficial de los cordones y b) Forma de los ripples de cada cordón. 
Las Figuras 5 y 6 muestran imágenes de las macrografías transversales y longitudinales, respectivamente. La Tabla 2 reporta las dimensiones de la geometría de los cordones (ancho y sobre-espesor) y la distancia entre valles y las penetraciones de los cordones. En dichas imagen y tabla se puede observar que la distancia entre valles del perfil de penetración disminuye conforme aumenta el tiempo del ciclo caliente. Además, se puede observar un máximo de penetración para la condición SC3 (1,84 mm). Es decir, el perfil de penetración aumenta desde la probeta SC1 hasta la SC3 y luego disminuye en SC4.
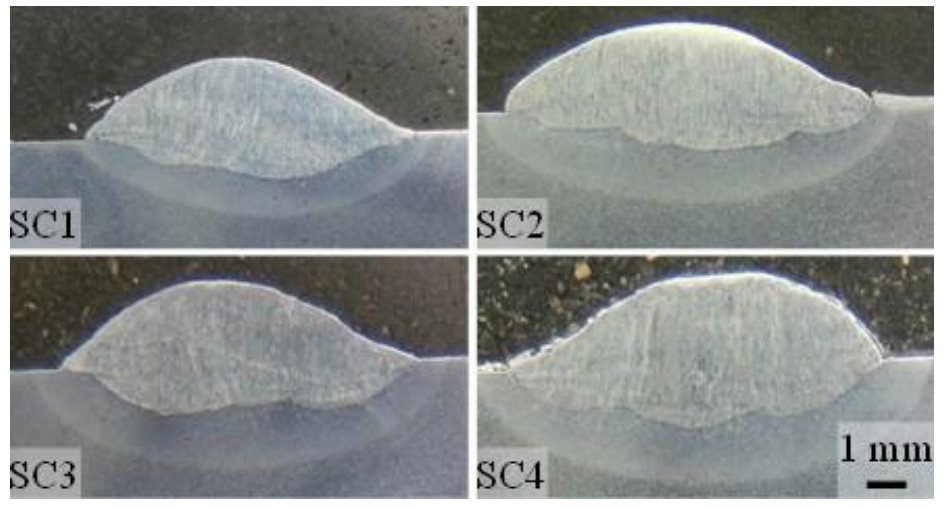

Figura 5: Macrografías transversales.
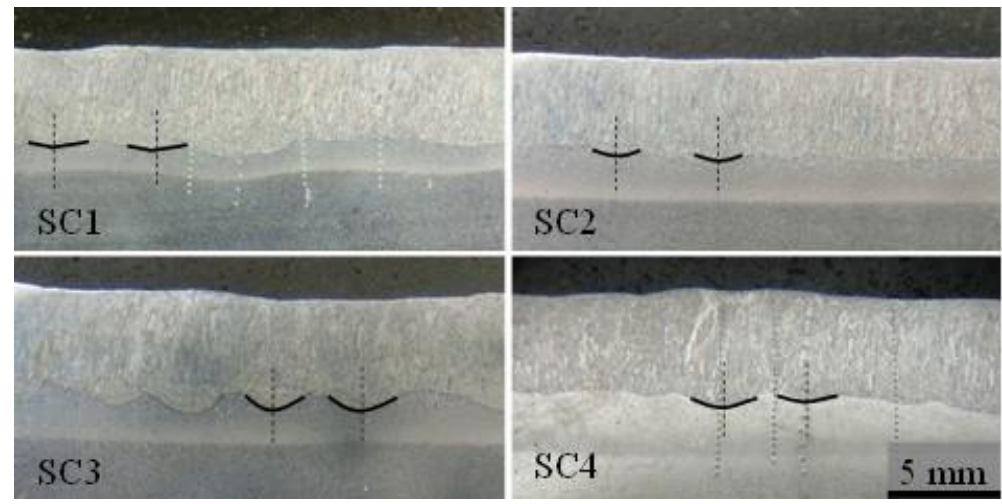

Figura 6: Macrografías longitudinales.

Tabla 2: Dimensiones geométricas de los cordones.

\begin{tabular}{l|l|l|l|l|l}
\hline & \multicolumn{2}{|l|}{ TRANSVERS } & & \multicolumn{2}{l}{ LONGITUDINAL } \\
\hline CUPON & ANCHO & SOBRE-ESPESOR & PENET 1 & PENET 2 & DISTANCIA ENTRE VALLES \\
\hline & {$[\mathrm{mm}]$} & {$[\mathrm{mm}]$} & {$[\mathrm{mm}]$} & {$[\mathrm{mm}]$} & {$[\mathrm{mm}]$} \\
\hline SC1 & 9,2 & 2,9 & 0,3 & 0,56 & 4,0 \\
\hline SC2 & 9,8 & 2,7 & 0,3 & 0,62 & 3,8 \\
\hline SC3 & 10,8 & 2,4 & 0,9 & 1,84 & 3,5 \\
\hline SC4 & 11,2 & 2,3 & 0,7 & 1,2 & 3,2 \\
\hline
\end{tabular}

La Figura 7 muestra las macrografías de las diferentes condiciones en estudio. Para revelar y cuantificar la microestructura del metal de soldadura y de la zona afectada por el calor se utilizaron las técnicas descriptas $[7,8]$. En dicha imagen se pueden observar las diferentes zonas que caracterizan a los cupones soldados: la chapa base, la zona afectada por el calor y los granos columnares, conformando el metal de soldadura. Además, se observan diferentes direcciones preferenciales de crecimiento y renucleación al aumentar o disminuir los tiempos de los ciclos calientes y fríos, respetivamente [9]. Es decir, para las probetas soldadas con menores tiempos del ciclo caliente (con menor calor aportado) se aprecian tres direcciones de crecimiento: una inferior (adyacente a la chapa base), una central y una superior. Cada una de éstas con una dirección particular. Por otro lado, para las probetas soldadas con mayores tiempos del ciclo caliente (con mayor calor 
aportado) se observan dos direcciones de crecimiento: la inferior y la superior. Sin embargo, la zona de renucleación y crecimiento superior se ubica cada vez más superficialmente a medida que aumenta el calor aportado.
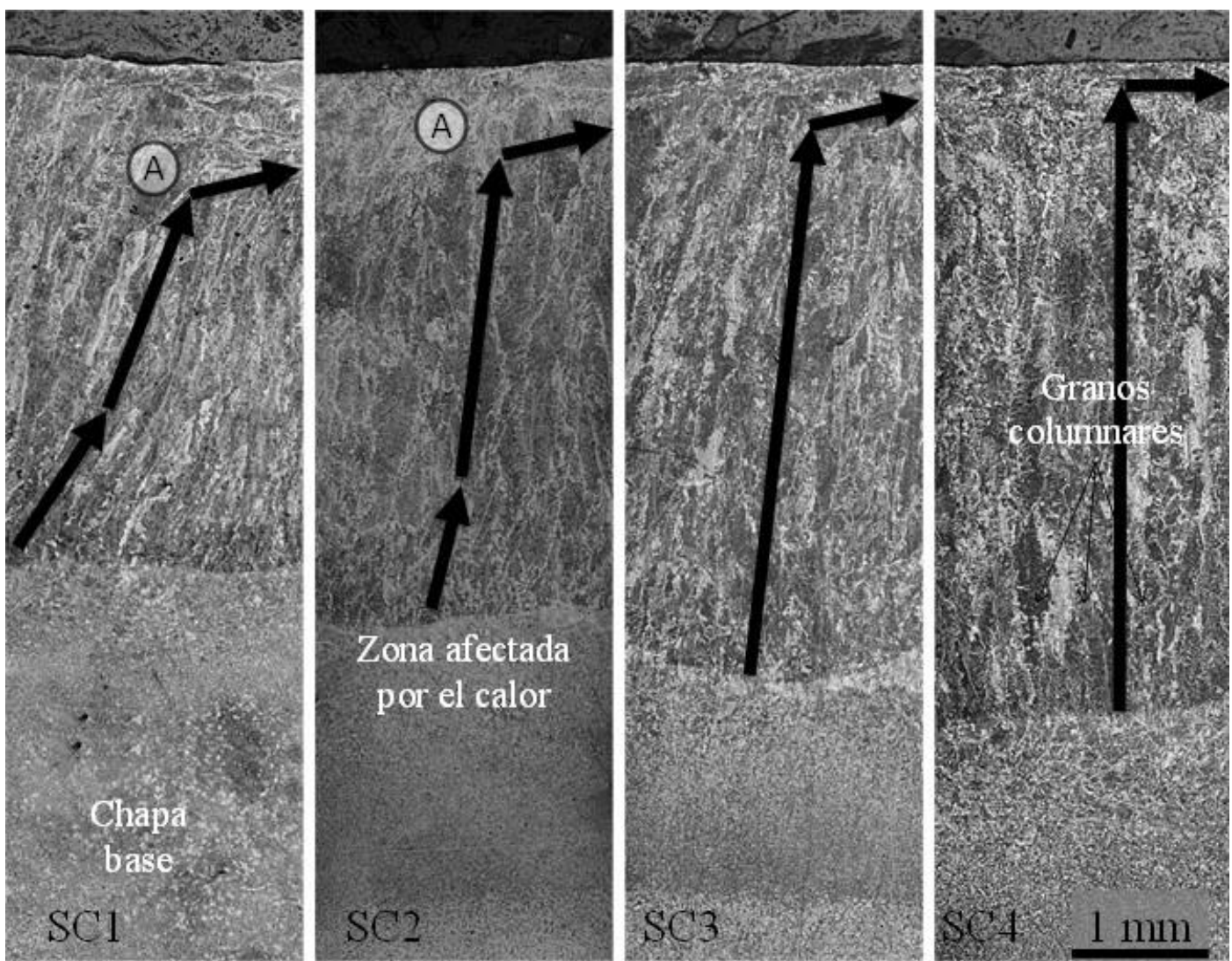

Figura 7: Macrografías de los diferentes cupones. Siendo las zonas A: zonas de renucleación.

La Tabla 3 muestra los valores promedios del ancho del grano columnar, tamaño de grano grueso y fino de la zona afectada por el calor para cada condición. En dicha tabla se puede observar que los tamaños de grano grueso y fino aumentan al aumentar el tiempo del ciclo caliente. De la misma manera, el ancho promedio de los granos columnares del metal de soldadura aumenta al aumentar el calor aportado.

Tabla 3: Tamaño promedio del grano columnar y de la zona afectada por el calor.

\begin{tabular}{l|l|l|l|l}
\hline & \multicolumn{2}{|l|}{ ZAC } & MS \\
\hline CUPON & TGG & TGF & TT & AGC \\
\hline & {$[\mathrm{mm}]$} & {$[\mathrm{mm}]$} & {$[\mathrm{mm}]$} & {$[\mu \mathrm{m}]$} \\
\hline SC1 & 0,61 & 0,72 & 1,33 & 111 \\
\hline SC2 & 0,73 & 1,17 & 1,90 & 173 \\
\hline SC3 & 0,86 & 1,24 & 2,10 & 187 \\
\hline SC4 & 1,82 & 3,36 & 2,49 & 193 \\
\hline
\end{tabular}

Siendo: ZAC: Zona Afectada por el Calor; RGG: Tamaño de Grano Grueso; TGF; Tamaño de Grano Fino; TT: Tamaño Total; MS: Metal de Soldadura; AGC: Ancho de Grano Columnar.

La Figura 8 muestra la microestructura obtenida del cupón SC1. En dicha imagen se puede observar e identificar las diferentes morfologías de ferrita, características en los depósitos de soldadura de estos materiales $[10,13]$. Después de la solidificación, el acero muestra la característica estructura de solidificación celular-dendrítica consistente en granos columnares y anchos de austenita y una fina red celular dentro de los granos, como se mencionó anteriormente. En el caso de soldadura de aceros al C-Mn o de baja aleación hay 
algunas dificultades con la nomenclatura para los productos de la descomposición de la austenita, que se deriva principalmente de observaciones morfológicas más que de detalles de los mecanismos de transformación [11].

La microestructura obtenida al enfriar el metal de soldadura desde el líquido hasta la temperatura ambiente se denomina estructura primaria o "como soldada". Esta consiste de ferrita alotriomórfica $\alpha$, ferrita con placas laterales o Widmanstatten $\alpha \mathrm{w}$, ferrita acicular $\alpha \mathrm{a}$ y las llamadas microfases, que pueden incluir pequeñas cantidades de martensita, austenita retenida o perlita. La bainita también se puede encontrar en ciertos depósitos de soldadura [12].

En el enfriamiento debajo de la temperatura Ac3, la ferrita nucleará inicialmente en las esquinas y bordes de grano austeníticos, ya que estos sitios generalmente proveen la menor barrera energética para la nucleación. La ferrita crecerá con una cinética parabólica dentro de la austenita detrás de un frente planar incoherente [13] llevando a la formación de alotriomorfos en los bordes de grano. La ferrita alotriomórfica también suele llamarse ferrita poligonal.

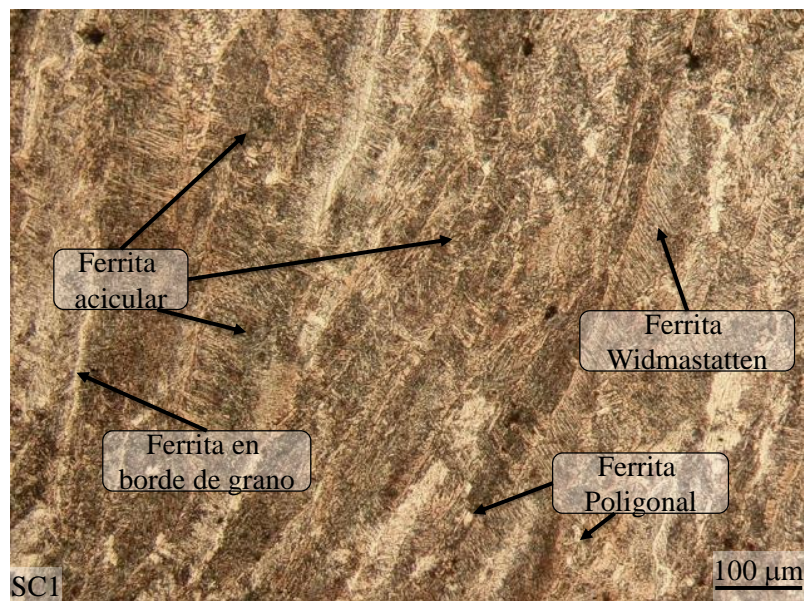

Figura 8: Microestructura del cupón SC1.

La Tabla 3 y la Figura 9 muestran los valores de los barridos de dureza y los promedios para los diferentes cupones. Las mediciones se realizaron según norma ASTM E384 [14]. En dichas tabla e imagen se puede observar la influencia del procedimiento de soldadura. Al aumentar, el tiempo del ciclo caliente la dureza promedio disminuyo desde los 233 hasta los $194 \mathrm{H}_{\mathrm{V} 1}$. Además, Los valores de los barridos de dureza disminuyeron desde la superficie hasta la zona afectada por el calor.

Tabla 4: Barridos de dureza de las diferentes condiciones.

\begin{tabular}{l|l|l|l|l}
\hline DISTANCIA & SC1 & SC2 & SC3 & SC4 \\
\hline$[\mathrm{mm}]$ & {$\left[\mathrm{H}_{\mathrm{V} 1}\right]$} & {$\left[\mathrm{H}_{\mathrm{V} 1}\right]$} & {$\left[\mathrm{H}_{\mathrm{V} 1}\right]$} & {$\left[\mathrm{H}_{\mathrm{V} 1}\right]$} \\
\hline 0,25 & 242,0 & 209,6 & 207,0 & 193,6 \\
\hline 0,50 & 236,3 & 223,0 & 209,6 & 203,0 \\
\hline 0,75 & 236,0 & 229,6 & 210,0 & 203,3 \\
\hline 1,00 & 239,6 & 227,0 & 217,0 & 207,0 \\
\hline 1,25 & 248,0 & 227,6 & 222,6 & 207,3 \\
\hline 1,50 & 242,6 & 230,0 & 212,0 & 202,6 \\
\hline 1,75 & 246,6 & 225,3 & 203,0 & 204,0 \\
\hline 2,00 & 229,6 & 224,3 & 213,6 & 206,0 \\
\hline 2,25 & 228,3 & 221,0 & 213,3 & 199,3 \\
\hline 2,50 & 227,6 & 211,6 & 207,6 & 192,3 \\
\hline 2,75 & 215,3 & 211,6 & 206,3 & 192,6 \\
\hline 3,00 & 199,0 & 212,3 & 205,0 & 193,0 \\
\hline
\end{tabular}




\begin{tabular}{l|l|l|l|l}
\hline 3,25 & & 199,6 & 206,3 & 185,0 \\
\hline 3,50 & & & 200,3 & 178,3 \\
\hline 3,75 & & & 190,6 & 176,3 \\
\hline 4,00 & & & & 166,0 \\
\hline Promedio & 233 & 220 & 208 & 194 \\
\hline
\end{tabular}

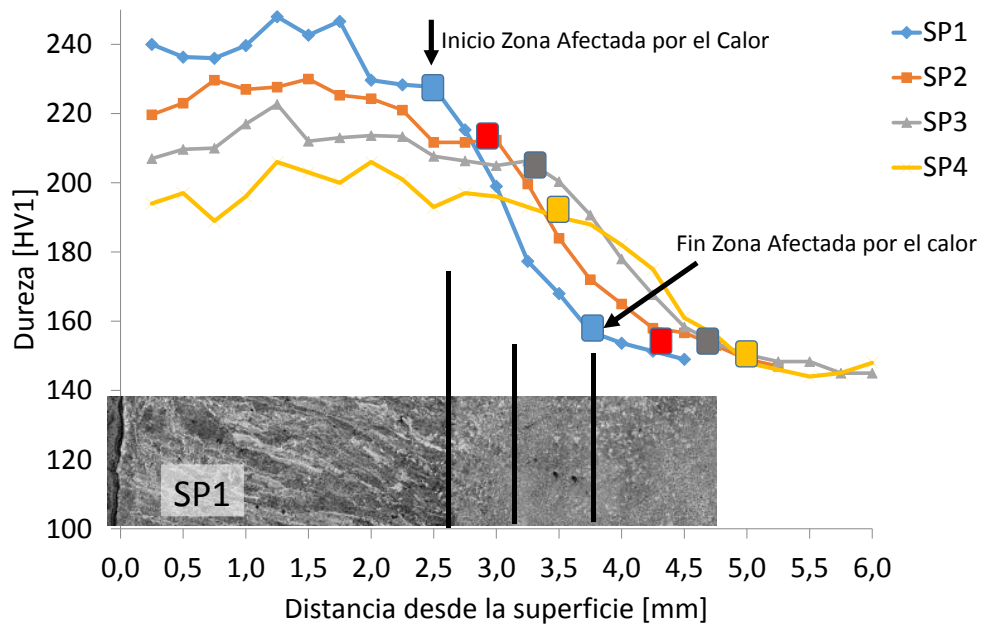

Figura 9: Barridos de dureza desde la superficie.

\section{DISCUSIÓN}

En la Figura 4 se puede observar que el nivel de salpicaduras generado durante la transferencia fue disminuyendo de SC1 a SC4. Esto podría estar asociado a los tiempos de los ciclos fríos y calientes, tiempos de transferencia spray y arco corto, respectivamente. Está reportado en la literatura [15] que el modo de transferencia spray está caracterizado por una deposición de material en pequeñas gotas, una mayor penetración y un bajo nivel de salpicaduras, respecto a otros modos de transferencia. En este sentido, al disminuir el tiempo de transferencia del arco corto y al aumentar el tiempo de transferencia spray la incidencia de éste último modo de trasferencia es mayor generando un menor nivel de salpicaduras. Además, en dicha imagen se puede observar que el ancho o separación de los ripples (ondulaciones) se incrementó con el aumento del tiempo de pulso de spray. Esto estaría asociado a que cada uno de los ripples generados durante la conformación del cordón responde, principalmente, al volumen de material depositado durante dicho modo el cual aumentó con el incremento del ciclo de spray.

En las macrografías transversales y longitudinales mostradas en las Figuras 5 y 6 , se puede observar la geometría y el perfil de penetración obtenido en los diferentes cupones, asociado a los ciclos de soldadura (caliente y frio). En la Tabla 2 se reporta que el ancho y el sobre espesor de los cordones aumenta y disminuye, respectivamente desde el cupón SC1 al SC4. Además, aumentando el tiempo del ciclo spray aumenta el perfil de penetración (indicados como penetración 1 y 2) y el promedio de la distancia entre valles disminuye. Una de las características principales en las soldaduras realizadas con control de onda Superpulso $[16,17]$ es la de obtener una profundidad de penetración variable dependiente de los parámetros eléctricos, los tiempos de cada ciclo y de la velocidad de soldadura. Al aumentar el tiempo de transferencia spray, con parámetros eléctricos más altos respecto al ciclo de arco corto, aumenta la temperatura de la pileta líquida generando cordones más anchos, de menor altura (sobre-espesor) pero con una mayor penetración. En este sentido, al mantener constante los parámetros eléctricos de cada ciclo y la velocidad de soldadura, aumentando el tiempo del ciclo caliente (spray), la distancia entre valles (zonas de mayor penetración) disminuye.

En un sentido más preciso, la variación en la geometría del cordón de soldadura debido a los cambios en los parámetros del proceso puede explicarse por las modificaciones en la dimensión y forma de la pileta líquida de la soldadura.

Zacharia et al. [18] han declarado que la dimensión y la forma de la pileta de soldadura se controlan críticamente mediante el efecto individual y combinado de varios factores como el mecanismo de flujo de fluido, la temperatura de la gota, la temperatura del arco, frecuencia de transferencia de la gota, el tamaño y aceleración de la gotita. 
Está bien establecido que estos factores son el resultado de la variación en los diferentes parámetros del proceso. El mecanismo de flujo de fluido, que es un producto de diferentes fuerzas motrices, juega un papel determinante en el control de la forma de la pileta líquida de soldadura. La magnitud y dirección del flujo de metal fundido en la pileta de soldadura puede alterar significativamente las condiciones locales de transferencia de calor y, en consecuencia, la geometría de la zona de fusión. Las fuerzas motrices para el flujo de fluido en la pileta de soldadura se dividen en dos grandes categorías: los términos de movimiento de volumen que incluyen las fuerzas de flotación (térmica / gravedad) y las fuerzas electromagnéticas y las fuerzas de corte y presión que actúan sobre la superficie de la pileta de soldadura debido al gradiente de tensión superficial y a la curvatura superficial [18]. El flujo del fluido en la pileta de soldadura también está influenciado por la presión del arco debido al plasma, pero en este caso debido a la baja corriente se considera despreciable [19].

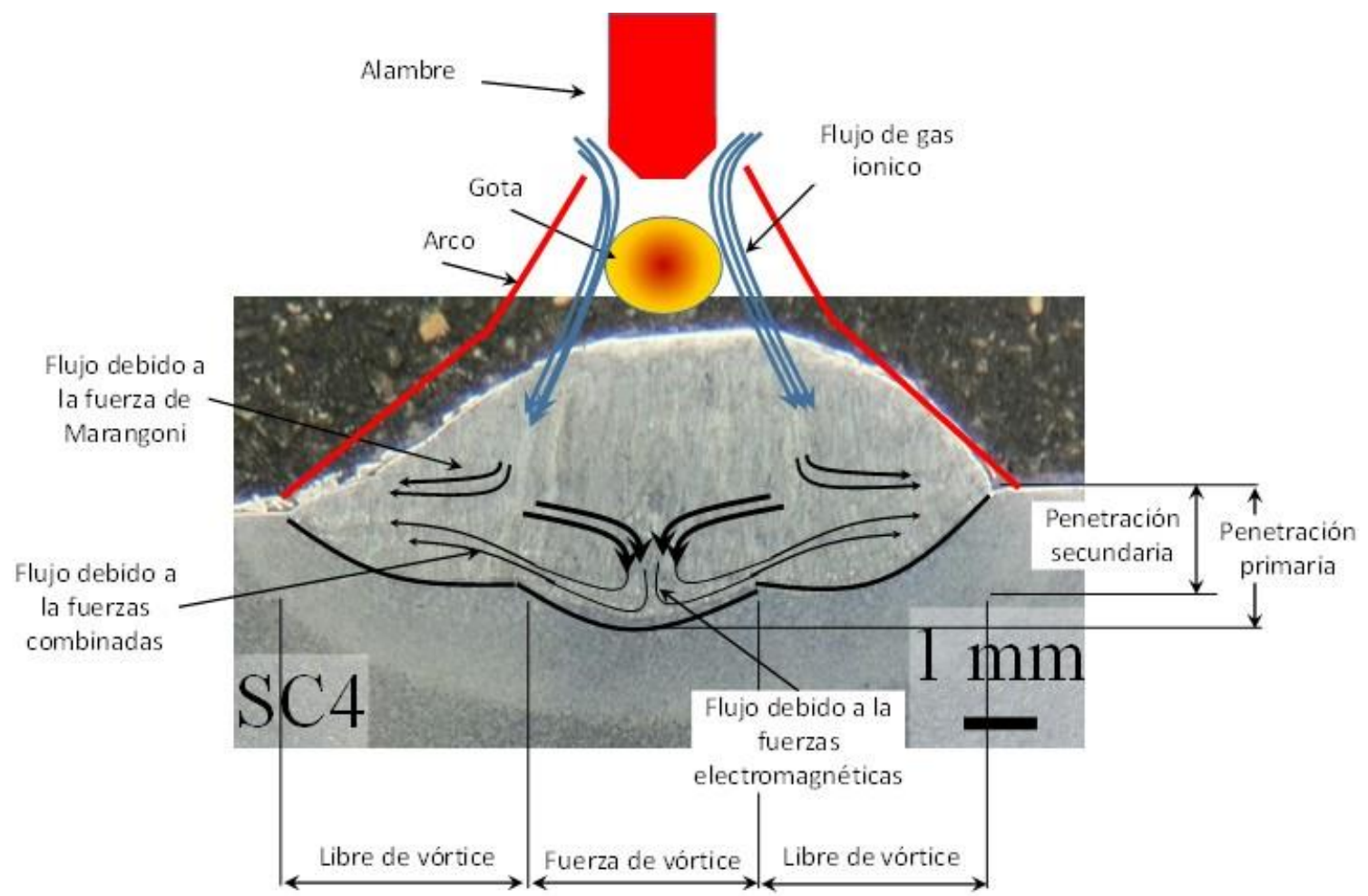

Figura 10: Esquema del efecto del flujo de fluido turbulento inducido en la pileta de soldadura por el resultado combinado de diferentes fuerzas de accionamiento sobre la soldadura

En el presente estudio, el aumento de la corriente media (condiciones soldadas con mayor tiempo de spray) ha causado un aumento en la fuerza de Lorentz dentro de la pileta de soldadura. Tal fuerza de Lorentz elevada desarrollada en la pileta de soldadura aumenta el movimiento hacia abajo, también conocido como movimiento de vórtice forzado [18], del fluido fundido y transmite calor directamente al fondo de la pileta de soldadura que conduce a una penetración primaria más profunda.

Además, es cierto que la fuerza de Marangoni y la fuerza de Lorentz actuaron juntas en el campo de flujo para controlar el área de la pileta de soldadura efectiva. Debido al aumento de la corriente media (condiciones soldadas con mayor tiempo de spray), la temperatura superficial de la pileta de soldadura aumenta, y Kou y Wang [20] ya han informado de que el aumento de temperatura disminuye la tensión superficial, lo que conduce finalmente a un gradiente de temperatura de tensión superficial negativo $\delta \gamma / \delta \mathrm{T}$ ). Más negativo $\delta \gamma$ / $\delta \mathrm{T}$ empuja el líquido fundido en la dirección hacia el exterior (mayor ancho).

La Figura 7 muestra, con bastante detalle el modo de solidificación de los diferentes cordones soldados. En ella, se puede identificar las diferentes zonas: la chapa base, la zona afectada por el calor y los granos columnares mostrando el patrón de solidificación. Además, se observan diferentes direcciones preferenciales de crecimiento de los granos columnares dependientes de los tiempos de los ciclos calientes y fríos. Comparando los cupones de soldadura desde SC1 a SC4 se puede observar que los granos columnares crecen en direcciones cada vez más verticales. Este fenómeno está asociado con la dirección de máxima extracción de calor. Al aumentar el tiempo del ciclo caliente, disminuyendo el tiempo del ciclo frio, manteniendo la veloci- 
dad de soldadura constante, la dirección de máxima extracción de calor se va haciendo cada vez mes vertical. En la literatura se reporta que las mayores temperaturas en el sólido se ubican sobre la línea central inmediatamente detrás de la pileta líquida, produciendo una transferencia de calor menos eficiente y resultando en un gradiente térmico mínimo a lo largo de esa dirección. Al mismo tiempo la velocidad de crecimiento del sólido es máxima a lo largo de la línea central [21]. De lo dicho hasta aquí también se desprende el concepto del crecimiento competitivo en los materiales policristalinos, donde cada cristal tiene una orientación cristalina distinta. De esta forma los cristales que tengan una dirección de crecimiento fácil $\langle 100\rangle$ mejor orientada respecto de la dirección del máximo gradiente térmico, crecerán selectivamente respecto de los que tengan su dirección $\langle 100\rangle$ peor orientada. Como se ve en la figura 7 es a menudo necesario que los cristales cambien de orientación durante el crecimiento a fin de continuar creciendo en la dirección del máximo gradiente térmico, que va cambiando con el tiempo debido a que la fuente está en movimiento. Esto produce una curvatura de los granos, que se produce por una ramificación lateral de la subestructura de solidificación [22]. Dado que la dirección cristalina no se modifica, los granos que se van curvando se desorientan cada vez más de su dirección de crecimiento fácil debido al continuo movimiento de la fuente y la ramificación lateral se hace cada vez más dificultosa, por lo que los cristales que estaban creciendo según una dirección $\langle 100\rangle$ no la puedan mantener en el tiempo. Para mantener la continuidad de crecimiento, los cristales que solidifican deben cambiar la orientación y esto requiere de una re-nucleación a lo largo de una nueva dirección <100> más apropiada $[9,23]$.

Por otro lado, de acuerdo a lo mostrado en la Figura 7 y a lo reportado en la Tabla 3 se observa que la re-nucleación superior se genera cada vez más cerca de la superficie de cada cordón y que el tamaño de grano columnar crece de SC1 a SC4. Estos fenómenos podrían estar asociados al mayor tiempo que tienen los granos columnares en crecer en forma vertical incrementando su ancho al aumentar el tiempos del ciclo spray. Además, las diferentes áreas de la zona afectada por el calor (zona de grano grueso y fino) crecen al aumentar el tiempo del ciclo spray asociado al mayor calor aportado.

En la Figura 8 se observa la microestructura del cupón SC1. Dicha microestructura es representativa de todos los cordones soldados. En la misma se pueden identificar las diferentes morfologías de ferrita: ferrita acicular, en borde de grano, poligonal y Widmanstatten.

En la Tabla 3 y la Figura 9 se pueden observar los perfiles de dureza desde la superficie hasta la chapa base y los valores promedios para cada condición. En términos generales, los barridos de dureza para todas las condiciones disminuyeron levemente desde la superficie hasta la chapa base. Por otro lado, se puede observar que los valores promedios de dureza disminuyen desde SC1 a SC4 (233 a $194 \mathrm{H}_{\mathrm{V} 1}$ ). Esto estaría relacionado principalmente con el aumento de la ferrita primaria debido al aumento de tamaño de grano austenítico y a la menor velocidad de enfriamiento [24, 25].

\section{CONCLUSIONES}

En este trabajo se estudió el efecto de los tiempos de los ciclos en soldaduras con control de onda Superpulso. El aumento del tiempo del ciclo caliente (spray) y la disminución del tiempo del ciclo frio (arco corto) generó las siguientes conclusiones:

Una disminución del nivel de salpicaduras. La geometría de los cordones cambió. Se evidenció un aumento del ancho, una disminución del sobre-espesor de los cordones y una mayor penetración (variable) con una disminución de la distancia entre valles. La dirección de crecimiento de los granos columnares aumenta hasta ser casi vertical. Dichos granos columnares crecen longitudinalmente generando zonas de renucleación y cambio de dirección de solidificación cada vez más superficiales. El ancho de dichos granos columnares aumenta. Los tamaños de las diferentes áreas de la zona afectada por el calor aumentan. Finalmente, la dureza de las diferentes condiciones de soldadura disminuye, controlado principalmente por el tamaño del grano columnar.

Es interesante el estudio de soldaduras con procesos modernos Superpulsos ya que al modificar los tiempos de los ciclos caliente y frío se controlan: la geometría del cordón de soldadura, la microestructura, el tamaño del grano columnar y su resistencia mecánica, entre otras cosas, factores interesantes en aplicaciones de pasadas de raíz y en soldaduras fuera de posición.

\section{AGRADECIMIENTOS}

Los autores del presente trabajo desean agradecer a CONARCO-ESAB Argentina, por las facilidades para realizar las soldaduras; la Universidad Nacional de Lomas de Zamora, a la Agencia Nacional de Promoción Científica y Tecnológica y al Ministerio de Educación por el apoyo financiero. 


\section{BIBLIOGRAFÍA}

[1] PRAVEEN, P., YARLAGADDA, P., KANG, M, “Advancements in pulse gas metal arc welding”, Journal of Materials Processing Technology, v. 164-165, pp. 1113-1119, 2005.

[2] RADICI, E., "Soldadura en sistemas de escapes de automóviles con proceso MIG - MAG aristo superpulse", Soldar Conarco, v. 134, pp. 17-29, 2011.

[3] PONOMAREV, V., SCOTTI A., SILVINSKIY A., AL-ERHAYEM, O., "Atlas of MIG/MAG welding metal transfer modes", In: International Institute of Welding Conference, Doc. No. XII-1771 to 1775, pp. 1-5, Bucharest, 2003.

[4] KURSEN, T., "Effect of the GMAW and GMAW-P welding processes on microstructure, hardness, tensile and impact strength of AISI 1030 steel Joint fabricated by ASP316L austenitic stainless steel filler metal", Archives of Metallurgy and Materials, v. 56, n. 4, pp. 955-965, 2011.

[5] KENT, L., "Evaluation of advanced gas metal arc welding and distortion mitigation techniques for thin panel steel and aluminum structure”, In: BMT Fleet Technology - Final Report, 2008.

[6] AWS A5.18/A5.18M, Specification for Carbon Steel Electrodes and Rods for Gas Shielded Arc Welding, 2005.

[7] PARGETER, R., DOLBY, R., "Guidelines for Classification of Ferritic Steel Weld Metal Microstructural Constituents Using the Light Microscope", In: International Institute of Welding Conference, IIW Doc No. IX-1377 to 1385 , pp. 1-7, 1985.

[8] ASTM E407, Standard Practice for Micro-etching Metals and Alloys, 2007.

[9] EARSTERLING, K., Introduction to the Physical Metallurgy of Welding, 1 ed., London, Butter-worths, 1983.

[10] AWS, Welding Handbook, Welding Process, 8th edition, v. 2, Miami, 1991.

[11] ASM, Metals Handbook, Metallography and Microstructures, 9th edition, v. 9, Miami, 1992.

[12] KOU, S. Welding Metallurgy, 1 ed., New Jersey, Wiley-Interscience Publication, 2002.

[13] CHADWICK, G. A., Metallography of Phase Transformations, 1 ed., New York, Russak and Company, 1972.

[14] ASTM E384, Standard Test Method for Knoop and Vickers Hardness of Materials, 2011.

[15] KOU, S., SUN, D., "Fluid flow and weld penetration in stationary arc welds. Metall.", Metallurgical Transaction A, v. 16A, pp. 203-213, 1985.

[16] LYTTLE, K., "Metal Cored Wires: Where Do They Fit in Your Future?", Welding Journal, v. 75, n. 10, pp. 35-38, 1996.

[17] SEN, M., MUKHERJEE, M., PAL, T., "Evaluation of Correlations between DP-GMAW Process Parameters and Bead Geometry", Welding Journal, v. 94, pp. 265-279, 2015.

[18] ZACHARIA, T., ERASLAN, A., AIDUN, D., et al., "Three-dimensional transient model for arc welding”, Metallurgical Transactions B, v. 20, n. 5, pp. 645-659, 1989.

[19] LIN, M., EAGER, T., "Influence of arc pressure on weld pool geometry", Welding Journal, v. 64, n. 6, pp. 163-169, 1985.

[20] KOU, S., WANG, Y., "Weld pool convection and its effect", Welding Journal, v. 65, n. 3, pp. 63-70, 1986.

[21] DAVIES, G., GARLAND, J., "Solidification structures and properties of fusion welds", International Metallurgical Review, v. 20, pp. 83-106, 1975.

[22] BHADESHIA, H. K. D. H., SVENSSON, L. E. "Mathematical Modelling of Weld Phenomena", 1993, Institute of Materials, London.

[23] SAVAGE W., "Solidification, segregation and weld imperfections", Welding in the World, v. 18, n. 5/6, pp. 89-112, 1980.

[24] NAKAGAWA, H., KATOH, M., MATSUDA, F., et al., "Crystallographic Anisotropy of Columnar Zone in Aluminum Weld Metal", Transactions of the Japan Welding Society, v. 2, n. 1, pp. 10-20, 1971.

[25] MODENESI, P., MARQUES, P., SANTOS, D., "Soldabilidade dos AçosTransformáveis (Apostila)", Departamento de Engenharia Metalúrgica e de Materiais, Belo Horizonte, 2004.

[26] EVANS, G., BAILEY, N., Metallurgy of Basic Weld Metal, 1ed., Woodhead Publishing, 1997. 